\title{
A New Branch and Bound Algorithm for the Cyclic Bandwidth Problem
}

\author{
Hillel Romero-Monsivais, Eduardo Rodriguez-Tello, and Gabriel Ramírez \\ CINVESTAV-Tamaulipas, Information Technology Laboratory. \\ Km. 5.5 Carretera Victoria-Soto La Marina, 87130 Victoria Tamps., Mexico \\ \{hromero, ertello, grtorres\}@tamps.cinvestav.mx
}

\begin{abstract}
The Cyclic Bandwidth problem (CB) for graphs consists in labeling the vertices of a guest graph $G$ by distinct vertices of a host cycle $C$ (both of order $n$ ) in such a way that the maximum distance in the cycle between adjacent vertices in $G$ is minimized. The CB problem arises in application areas like VLSI designs, data structure representations and interconnection networks for parallel computer systems.

In this paper a new Branch and Bound (B\&B) algorithm for the CB problem is introduced. Its key components were carefully devised after an in-depth analysis of the given problem. The practical effectiveness of this algorithm is shown through extensive experimentation over 20 standard graphs. The results show that the proposed exact algorithm attains the lower bounds for these graphs (of order $n \leq 40$ ) expending a reasonable computational time.
\end{abstract}

Key words: Branch and Bound, Cyclic Bandwidth Problem

\section{Introduction}

The Cyclic Bandwidth problem (CB) was first stated by Leung et al. in 1984 [1] in relation with the design of a ring interconnection network for a set of computers $(V)$ whose communication pattern was described by a graph $G(V, E)$ where $\{u, v\} \in E$ if computer $u$ communicates with computer $v$. They were interested in finding an arrangement of these computers on a cycle so that every message sent can arrive at its destination in at most $k$ steps. The CB problem arises also in other application areas like VLSI designs [2], data structure representations [3], code design [4] and interconnection networks for parallel computer systems [5].

The CB problem can be formally defined as follows. Let $G(V, E)$ be a finite undirected graph, where $V(|V|=n)$ defines the set of vertices and $E \subseteq V \times V$ $=\{\{u, v\} \mid u, v \in V\}$ is the set of edges. Given a bijective labeling function for the vertices of $G, \varphi: V \rightarrow\{1,2, \ldots, n\}$, the cyclic bandwidth (the cost) for $G$ with respect to the labeling $\varphi$ is defined as:

$$
B_{c}(G, \varphi)=\max _{(u, v) \in E}\left\{|\varphi(u)-\varphi(v)|_{n}\right\}
$$


where $|x|_{n}=\min \{|x|, n-|x|\}$ for $0<|x|<n$, which is called cyclic distance. Then the $\mathrm{CB}$ problem consists in finding a labeling $\varphi^{*}$, which minimizes $B_{c}\left(G, \varphi^{*}\right)$, i.e.,

$$
B_{c}\left(G, \varphi^{*}\right)=\min \left\{B_{c}(G, \varphi): \varphi \in \mathscr{L}\right\},
$$

where $\mathscr{L}$ is the set of all possible labelings.

For instance, consider the graph $G(V, E)$ depicted in Fig. 1(a), consisting of ten vertices with a labeling $\varphi$ given by the numbers shown inside each vertex. The cyclic distance between each pair of adjacent vertices $(u, v) \in E$ can be calculated using the expression $\min \{|\varphi(u)-\varphi(v)|, n-|\varphi(u)-\varphi(v)|\}$. These cyclic distances are represented by the numbers associated to the edges in the graph. For this particular labeling $\varphi, B_{c}(G, \varphi)=4$. On the other hand, if the labels 1 and 9 are exchanged a new labeling $\varphi^{\prime}$ is obtained (see Fig. 1(b)), which produces a smaller (better) cyclic bandwidth value $\left(B_{c}\left(G, \varphi^{\prime}\right)=3\right)$. The embedding of this new labeling of the graph $\mathrm{G}$ in a cycle is presented in Fig. 1(c) for illustrative purposes.

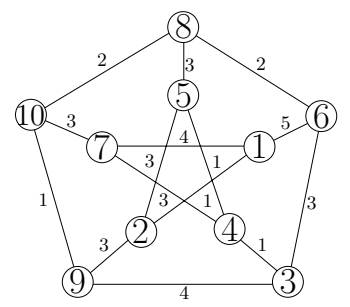

(a) Labeling $\varphi$

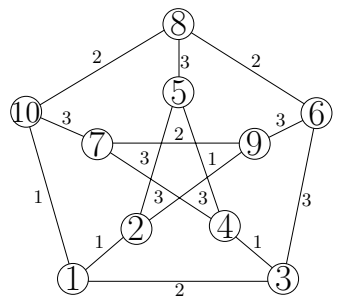

(b) Labeling $\varphi^{\prime}$

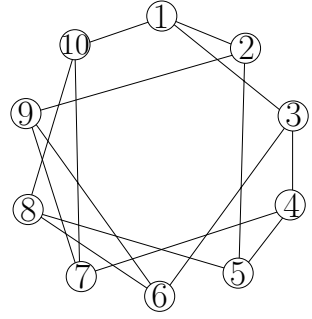

(c) Embedding $G$ in a cycle

Fig. 1. Example of a Cyclic Bandwidth problem instance.

It was demonstrated that finding the cyclic bandwidth is NP-complete for general graphs [6]. It is important to note that the $\mathrm{CB}$ problem is a natural extension of the well-known bandwidth minimization problem for graphs [7], which consists in embedding the vertices of a guest graph $G$ in a host path $P$ (both of order $n$ ) in such a way that the maximum distance in the path between adjacent vertices in $G$ is minimized.

In this paper, a new Branch and Bound (B\&B) algorithm for finding exact solutions for the $\mathrm{CB}$ problem for general graphs is presented. The proposed $\mathrm{B} \& \mathrm{~B}$ algorithm is based on a lowest-cost search strategy, i.e., branches having the lowest cost are explored while branches with higher cyclic bandwidth are cut. It implements the Hungarian method [8] for solving the problem of efficiently computing the cost of assigning every available label in a partial solution to each possible non-labeled vertex. The practical usefulness of the proposed B\&B is assessed experimentally using a test-suite, composed by 20 standard graphs belonging to 4 different classes. The computational results from this experiments 
show that our algorithm is able to attain the lower bounds for these graphs (of order $n \leq 40$ ) expending a reasonable computation time.

The remainder of this paper is organized as follows. In Sect. 2 a brief review is given to present some representative related work. The main components of the proposed B\&B algorithm are presented in detail in Sect. 3. Section 4 describes the experimental methodology used for assessing the practical usefulness of the proposed $\mathrm{B} \& \mathrm{~B}$ algorithm, while the computational results produced by these experiments are presented in Sect. 5. Finally, the conclusions of this work and some possible directions for future research are provided in Sect. 6 .

\section{Relevant Related Work}

In spite of its practical and theoretical importance, less attention has been paid to the CB problem with respect to other graph labeling problems. Up to now, most of the research on this important labeling problem has been devoted to the theoretical study of its properties with the aim of finding exact solutions for certain specific cases. Next, we present a brief review of these studies.

In 2002 Zhou [9] proposed a systematic method for obtaining lower bounds for the bandwidth and cyclic bandwidth problems in terms of some distanceand degree-related parameters of the graph. The main idea of this method is to relax the condition of embedding the graph $G$ on the host graph with the aid of a graphical parameter possessing some kind of monotonic property. This method has been demonstrated to be efficient when the parameters are chosen appropriately. The author concludes that this method yields a number of lower bounds for the ordinary and cyclic bandwidths. In both cases, it gives rise to new estimations, as well as improvements of some known results.

Later, de Klerk et al. [10] proposed two new semidefinite programming (SDP) relaxations of the bandwidth and cyclic bandwidth based on the quadratic assignment problem (QAP) reformulation. The bounds produced by this method were tested for some special graphs showing that they are tight for paths, cliques, complete bipartite graphs. However, these bounds are not tight for hypercubes, rectangular grids and complete $k$-level $t$-ary trees.

In 1995 Yuan and Zhou [11] demonstrated that for unit interval graphs, there exists a labeling which is simultaneously optimal for the following seven labeling problems: bandwidth, cyclic bandwidth, profile, cutwidth, modified cutwidth and bandwidth sum. Following this idea, in [12] Lam et al. made a characterization of graphs with equal bandwidth and cyclic bandwidth which includes planar graphs, triangulation meshes and grids with some specific characteristics.

To the best of our knowledge, there has been no previous research investigating Branch and Bound based algorithms for solving the CB problem in the case of general graphs. Therefore, this paper presents an original contribution in this field. 


\section{A New Branch and Bound Algorithm}

In this section, we present a new exact algorithm, based on Branch and Bound (B\&B), for solving the CB problem. This algorithm employs a lowest-cost search strategy, where branches having the lowest cost are explored while branches with higher cost are cut. The following subsections describe in detail the main components of our algorithm.

\subsection{Branching Strategy}

Every possible labeling $\varphi_{k}$, for $k=\{1,2,3, \ldots,(n-1) ! / 2\}$, must be tested. In our algorithm, the labelings are tested in lexicographic order, beginning with $\varphi_{1}=\{1,2,3, \ldots, n\}$ and ending with $\varphi_{(n-1) ! / 2}=\{n, n-1, n-2, \ldots, 1\}$. Each individual label in $\varphi_{k}$ is assigned to vertices, one at a time, producing three different sets: $V^{\prime}$ the set of already labeled vertices, $V_{k}^{\prime \prime}$ the set of unlabeled vertices which are adjacent to those in $V^{\prime}$ and $\varphi_{k}^{\prime \prime}$ the lexicographic order set of available labels.

The cyclic bandwidth (cost) $B_{c}\left(G, \varphi_{k}^{\prime}\right)$ for every particular partial labeling is computed considering $V^{\prime}$ in $G$. If all labels in $\varphi_{k}$ have been assigned, then a labeling $\varphi_{k}^{\prime}$ with a value $B_{c}\left(G, \varphi_{k}^{\prime}\right)$ smaller than the prefixed bound $b$ has been found. The algorithm then stores this new best solution in $\varphi^{\sigma}$.

According to Leung [1], the upper bound for the CB is $n / 2$, so at the beginning $b=n / 2$. When a labeling $\varphi^{\sigma}$ is found, the search retakes a new bound $b=B_{c}\left(G, \varphi^{\sigma}\right)-1$.

The algorithm performs a depth-first search, so if a certain label is assigned to a vertex and $B_{c}\left(G, \varphi_{k}^{\prime}\right)>b$, the branch is cut. Then, a backtrack to the parent label is applied and then the algorithm advances to the next child label. The search process stops when all labelings have been analyzed. A detailed description of the proposed B\&B method is summarized in Algorithm 1.

\subsection{Assignment Strategy}

Two important issues are considered when adding a new label to a partial solution $\varphi_{k}^{\prime}$ : the cost generated by that new label, and the lowest cost which can be produced by assigning the available labels $\varphi_{k}^{\prime \prime}$ to the set $V_{k}^{\prime \prime}$ of unlabeled vertices adjacent to those in $V^{\prime}$. The latter provides information allowing to know if the adjacent vertices can be labeled without exceeding the current bound $b$ in further iterations.

The Hungarian method is an algorithm which solves the assignment problem in polynomial time [8]. Our implementation employs this algorithm to compute the cost of assigning each label $l \in \varphi_{k}^{\prime \prime}$ to each possible vertex $v \in V_{k}^{\prime \prime}$. However, if the cost of an assignment becomes higher than the current bound $b$, then the evaluation of $\varphi_{k}$ is not completed.

For instance, consider the partial representation of a graph $G=(V, E)$ depicted in Fig. 2, which consists of five vertices labeled with the number shown within each vertex. Let $n=10$ and $b=3$ for this example. Since the vertices in 


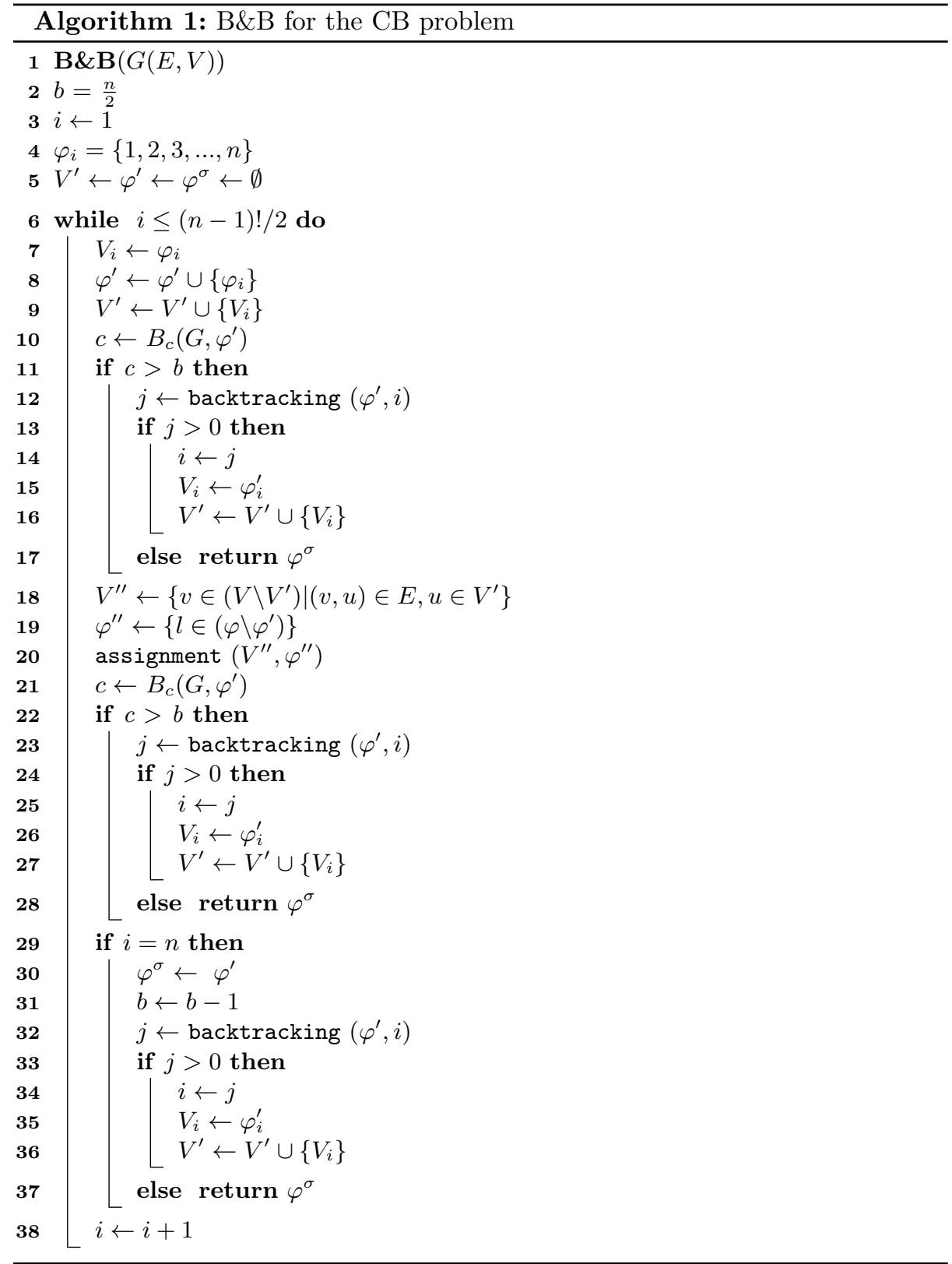

the set $V$ are taken following a lexicographic order, then the last labeled vertex was $V_{6} \leftarrow 5$, therefore $V^{\prime}=\left\{\ldots V_{5}, V_{6}\right\}, \varphi_{k}^{\prime}=\{\ldots, 3,5\}$ and $V_{k}^{\prime \prime}=\left\{V_{7}, V_{8}, V_{9}\right\}$. Suppose that $\varphi_{k}^{\prime \prime}=\{1,4,9,10\}$ and for this partial labeling $B_{c}\left(G, \varphi_{k}^{\prime}\right)<b$. Using the Hungarian method the costs of assigning the labels in $\varphi_{k}^{\prime \prime}$ to the vertices 


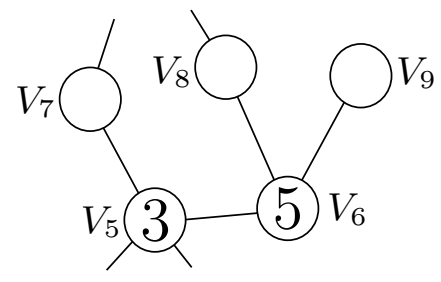

Fig. 2. Example of a partially labeled graph.

in $V_{k}^{\prime \prime}$ are estimated and presented in Table 1. From this table it is clear that $V_{7}$ and $V_{8}$ could be labeled without exceeding the current bound $b$ in further iterations. However, $B_{c}\left(G, \varphi_{k}^{\prime}\right) \leq b$ could not be guaranteed for $V_{9}$. In this case, the evaluation of $\varphi_{k}$ is stopped making a backtracking which produces the next possible labeling $\varphi_{k+1}$ in lexicographic order.

Table 1. Table assignment vertex-label

\begin{tabular}{llllr}
\hline & \multicolumn{5}{c}{$\varphi_{k}^{\prime \prime}$} \\
\cline { 2 - 5 }$V_{k}^{\prime \prime}$ & 1 & 4 & 9 & 10 \\
\hline$v_{7}$ & $\mathbf{2}$ & 1 & 5 & 3 \\
$v_{8}$ & 4 & $\mathbf{1}$ & 4 & 5 \\
$v_{9}$ & 4 & 1 & 4 & 5
\end{tabular}

After preliminary experimentation with this basic assignment strategy we have observed that it could be improved. The amelioration consists in also analyzing the assignments of the adjacencies between the vertices belonging to the set $V_{k}^{\prime \prime}$. Although it represents an additional computational effort, it permits to find at this step vertices producing a cost which exceeds the current bound $b$. The main advantage of this improved assignment strategy is that it is able in certain cases to stop the evaluation of $\varphi_{k}$ earlier than the basic assignment strategy, which reduces importantly the size of the search space.

\subsection{Backtracking Strategy}

A backtracking occurs if the cyclic bandwidth of the partial labeling $\varphi_{k}^{\prime}$ exceeds the current bound $b$ when its $i$-th label is added. This backtracking procedure is performed as described in Algorithm 2. First, the best element in the set $\varphi_{k}^{\prime \prime}$ of available labels is identified. It is the first label $l \in \varphi_{k}^{\prime \prime}$ which guarantees that the resulting cyclic bandwidth $B_{c}\left(G, \varphi_{k}^{\prime}\right)$ is lower than the current bound $b$ when this label is assigned to the $i$-th position of the partial labeling $\varphi_{k}^{\prime}$. If there is no such label meeting this condition, the $(i-1)$-th position of $\varphi_{k}^{\prime}$ is analyzed (a backtracking occurs), the set $\varphi_{k}^{\prime \prime}$ is updated and its best label is searched again. 
This process is repeated until the best label is found or the complete set $\varphi_{k}^{\prime}$ was analyzed.

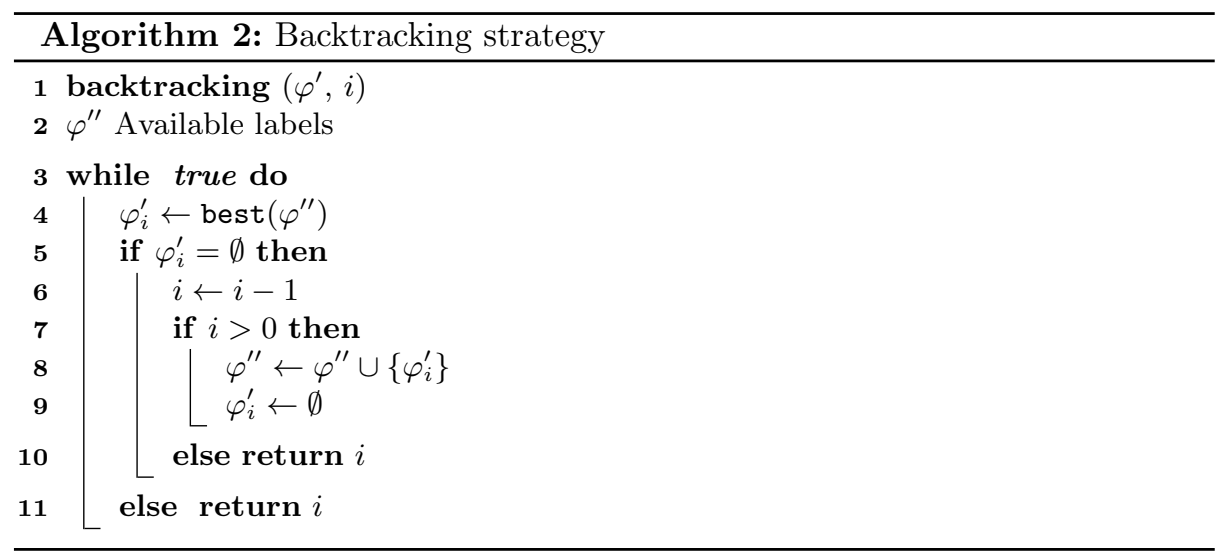

\section{Experimental Setup}

In order to assess the performance of the proposed $\mathrm{B} \& \mathrm{~B}$ algorithm introduced in Sect. 3 it was was coded in $\mathrm{C}$ and compiled with $g c c$ using the optimization flag -O3. It was run sequentially into a cluster composed of 4 processors Xeon X5650 equipped with six cores at $2.66 \mathrm{GHz}, 32 \mathrm{~GB}$ of RAM and Linux operating system.

Due to the deterministic nature of the algorithm, it was executed only one time over each of the selected benchmark instances. The maximum CPU time allowed for each execution was predefined to 36 hours.

\subsection{Compared Algorithms}

For the computational experiments two different versions of the B\&B algorithm described in Sect. 3 were implemented. The main difference between these two versions is the assignment strategy employed. The first one, denoted B\&B1, employs the basic assignment strategy, which employs the Hungarian method to solve the problem of finding the cost (cyclic bandwidth) of assigning each label $l \in \varphi_{k}^{\prime \prime}$ to each possible vertex $v \in V_{k}^{\prime \prime}$. The second version, called B\&B2, uses the improved assignment strategy which also analyzes the assignments of the adjacencies between the vertices belonging to the set $V_{k}^{\prime \prime}$ (see Sect. 3.2).

\subsection{Benchmark Instances and Performance Assessment}

As it was mentioned at the end of Sect. 2, there has been no previous research investigating neither exact nor approximate algorithms for solving the CB prob- 
Table 2. Characteristics of the test-suite used in the experiments. It consists of 20 instances representing 4 different classes of graphs.

\begin{tabular}{|c|c|c|c|c|}
\hline Graph & $|V|=n$ & $|E|=m$ & $E D=2 m / n(n-1)$ & $\mathcal{T}$ \\
\hline cycle20 & 20 & 20 & 0.105 & 1 \\
\hline cycle 25 & 25 & 25 & 0.083 & 1 \\
\hline cycle30 & 30 & 30 & 0.069 & 1 \\
\hline cycle35 & 35 & 35 & 0.059 & 1 \\
\hline$--\frac{\text { cycle } 40}{\text { grid }} \overline{5}-\frac{1}{4} 4$ & $--\frac{40}{20}-$ & $--\frac{40}{31}$ & $-----\frac{0.051}{0.163}$ & $-\frac{1}{4}--$ \\
\hline $\operatorname{grid} 5 \times 5$ & 25 & 40 & 0.133 & 5 \\
\hline $\operatorname{grid} 5 \times 6$ & 30 & 49 & 0.113 & 5 \\
\hline $\operatorname{grid} 5 \times 7$ & 35 & 58 & 0.097 & 5 \\
\hline $\operatorname{grid} 5 \times 8$ & 40 & 67 & 0.086 & 5 \\
\hline path20 & $\overline{2} 0^{-}$ & $1 \overline{9}$ & $0.100^{-}$ & $\overline{1}$ \\
\hline path25 & 25 & 24 & 0.080 & 1 \\
\hline path30 & 30 & 29 & 0.067 & 1 \\
\hline path35 & 35 & 34 & 0.057 & 1 \\
\hline- - $\frac{\text { path } 40}{\text { tree } 21}-$ & $-\frac{40}{21}-$ & $--\frac{39}{20}$ & $-----\frac{0.050}{0.095}$ & $-\frac{1}{3}-$ \\
\hline tree 25 & 25 & 24 & 0.080 & 4 \\
\hline tree31 & 31 & 30 & 0.065 & 4 \\
\hline tree33 & 33 & 32 & 0.061 & 4 \\
\hline tree35 & 35 & 34 & 0.057 & 4 \\
\hline
\end{tabular}

lem in the case of general graphs. There exists no standard test-suite reported in the literature for evaluating the performance of this kind of algorithms. For this reason a test-suite composed of 20 benchmark instances is proposed in this paper.

The test-suite comprises 4 different classes of standard graphs: cycles, grids, paths and perfect binary trees. All these instances have a number of vertices between 20 and 40 and are publicly available ${ }^{1}$. The characteristics of these instances are detailed in Table 2, where the first three columns indicate the name of the graph as well as its number of vertices and edges. The edge density $(2|E| /|V|(|V|-1))$ of each graph is depicted in Column 4, while the last column presents the theoretical lower bounds $\mathcal{T}$ reported in [10,13,12].

The criteria used for evaluating the performance of the compared B\&B algorithms are the same as those used in the literature [14,15]: the total number of partial solutions $\left(\varphi_{k}^{\prime}\right)$ examined during the search process, the total number of backtracking operations occurred and the expended CPU time in seconds. For these criteria smaller values are better.

\footnotetext{
${ }^{1}$ http://www.tamps.cinvestav.mx/ ertello/cbmp.php
} 


\section{Computational Results}

The experimental comparison between the two versions of the Branch and Bound algorithm introduced in this paper (B\&B1 and B\&B2) was performed over the benchmark instances described above and employing the same computational platform introduced in Sect. 4.

The results from this experiment are summarized in Table 3, which lists in the first column the name of the benchmark instance. For each compared algorithm this table presents: the total number of partial solutions $\varphi_{k}^{\prime}$ evaluated during the search process $\left(\# \varphi_{k}^{\prime}\right)$, the total number of backtracking operations occurred $(B)$, and the CPU time in seconds (time) expended for finding the optimal solution $\varphi *$. Instances marked with the symbol "- " indicate that they could not be solved within the maximum allowed time of 36 hours. Given that we are comparing exact algorithms, it is not necessary to report the cyclic bandwidth achieved as it is always the optimum value.

Table 3. Performance comparison between two different versions (B\&B1 and B\&B2) of the Branch and Bound algorithm proposed in Sect. 3.

\begin{tabular}{|c|c|c|c|c|c|c|}
\hline \multirow[b]{2}{*}{ Graph } & \multicolumn{3}{|c|}{ B\&B1 } & \multicolumn{3}{|c|}{ B\&B2 } \\
\hline & $\# \varphi^{\prime}$ & $B$ & time & $\# \varphi^{\prime}$ & $B$ & time \\
\hline cycle20 & 2331 & 2042 & 0.01 & 586 & 487 & 0.01 \\
\hline cycle 25 & 12900 & 11867 & 0.12 & 627 & 514 & 0.01 \\
\hline cycle30 & 88516701 & 81990664 & 1007.90 & 2223754 & 2089272 & 43.10 \\
\hline cycle35 & 465326343 & 431788129 & 4184.05 & 46183164 & 43443834 & 449.58 \\
\hline cycle40 & 397409592 & 372614337 & 4996.08 & 23409592 & 22614337 & 531.08 \\
\hline grid $5 \times \bar{x} 4$ & $-\overline{12} 3 \overline{2} 3 \overline{30}$ & $\overline{11} \overline{3} 4 \overline{7} 2$ & $\overline{0} . \overline{8} 2^{-}$ & $\overline{1} \overline{2} \overline{80}$ & $-\overline{17002}$ & $-\overline{0.16}$ \\
\hline $\operatorname{grid} 5 \times 5$ & 2674635 & 2517186 & 29.32 & 562911 & 532316 & 8.43 \\
\hline $\operatorname{grid} 5 \times 6$ & 144312826 & 135950146 & 3016.46 & 3253192 & 3099321 & 84.63 \\
\hline $\operatorname{grid} 5 \times 7$ & - & - & - & 18490735 & 17431435 & 426.32 \\
\hline $\operatorname{grid} 5 \times 8$ & - & 一 & - & 160963339 & 155466427 & 14144.13 \\
\hline path 20 & $\overline{6} 9 \overline{5}$ & $5 \overline{8} 5$ & $\overline{0} . \overline{0} 1^{-}$ & $\overline{559}$ & $\overline{457}$ & $\overline{0} . \overline{01}$ \\
\hline path25 & 19997 & 18023 & 0.07 & 2498 & 2227 & 0.01 \\
\hline path30 & 101821 & 93156 & 7.63 & 2847814 & 2513041 & 0.60 \\
\hline path35 & 74072 & 70352 & 0.83 & 10393 & 9815 & 0.13 \\
\hline path40 & - & - & - & 10045277883 & 9116835089 & 34023.25 \\
\hline tree- 21 & $1 \overline{4} 7 \overline{2} 3 \overline{2}$ & $\overline{13} 36 \overline{5} 1$ & $0 . \overline{4} 7^{-}$ & $\overline{6} 1 \overline{4} 83$ & $\overline{5} 6 \overline{6} \overline{44}$ & $\overline{0} . \overline{23}$ \\
\hline tree 25 & 223355577 & 204264652 & 763.27 & 28450274 & 26320726 & 110.92 \\
\hline tree31 & 707968183 & 663767447 & 3552.96 & 220801934 & 208577566 & 1240.82 \\
\hline tree33 & 2248823425 & 2121853157 & 10965.57 & 882035992 & 836064022 & 4588.00 \\
\hline tree35 & - & - & - & 16419099889 & 15388330578 & 11288.81 \\
\hline
\end{tabular}

Analyzing the data presented in Table 3 lead us to the following main observations. First, the most time-consuming algorithm is B\&B1, despite B\&B2 
employs the improved assignment strategy which demands an additional computational effort. This is possible because the improved assignment strategy used by $\mathrm{B} \& \mathrm{~B} 2$ is able to importantly reduce the size of the explored search space (see column $\left.\# \varphi_{k}^{\prime}\right)$.

Second, one observes that B\&B1 was not able to find a solution within the time limit of 36 hours for the following classes of graphs with $n \geq 35$ : grids, paths and perfect binary trees. On the contrary, B\&B2 found an exact solution for all the selected instances employing at most 9.45 hours and consistently a lower number of backtrack operations $(B)$ than $\mathrm{B} \& \mathrm{~B} 1$.

The comparison of the computational time expended by B\&B1 and B\&B2 is better illustrated in Fig. 3. The plot represents the studied instances (ordinate) against the CPU time in seconds expended by the two compared algorithms using a $\log _{10}$ scale (abscissa). From this graph one clearly observes that B\&B2 consistently expends much less CPU time than B\&B1.



Fig. 3. Consumed CPU time comparison between two different versions (B\&B1 and $\mathrm{B} \& \mathrm{~B} 2)$ of the proposed Branch and Bound algorithm.

\section{Conclusions and Further Work}

In this paper a new Branch and Bound (B\&B) algorithm for the Cyclic Bandwidth $(\mathrm{CB})$ problem was introduced. It is based on a lowest-cost search strategy which explores the branches having the lowest cost while it cuts the branches with higher cyclic bandwidth.

Two different versions of this B\&B algorithm, employing slightly different assignment strategies, were implemented. The main difference between these two versions is that the first one, denoted B\&B1, employs a basic assignment 
strategy based on the Hungarian method [8] for efficiently finding the cost of assigning every available label in a partial solution to each possible non-labeled vertex; while the second, called $\mathrm{B} \& \mathrm{~B} 2$, uses an improved assignment strategy which additionally analyzes the assignments of the vertices belonging to the set of unlabeled vertices adjacent to those in the set of already labeled vertices.

Given the lack in the literature of a standard test-suite for evaluating the performance of algorithms for solving the CB problem, a new benchmark composed of 20 instances, comprising 4 different classes of standard graphs (cycles, grids, paths and perfect binary trees), was proposed in this work.

A performance experimental comparison between the two versions of the Branch and Bound algorithm introduced in this paper (B\&B1 and B\&B2) was performed over the benchmark instances proposed. The results from this comparison show that B\&B2 consumes much less CPU time (up to $97 \%$ in some cases), because it analyzes fewer partial solutions before finding the lower bound of the instances. This gives us the opportunity to find exact solutions for graphs than B\&B1 was unable to solve.

This work opens up a range of possibilities for future research. Currently, we are interested in hybridizing our B\&B algorithm with some kind of metaheuristic in order to speed the convergence time of the resulting algorithm, inspired in the ideas presented in [15]. The parallelization of the B\&B algorithm introduced in this paper also represents an interesting issue for future work.

\section{Acknowledgments}

This research work was partially funded by the following projects: CONACyT 99276, Algoritmos para la Canonización de Covering Arrays; 51623 Fondo Mixto CONACyT y Gobierno del Estado de Tamaulipas.

\section{References}

1. Leung, J., Vornberger, O., Witthoff, J.: On some variants of the bandwidth minimization problem. SIAM Journal on Computing 13(3) (1984) 650-667

2. Bhatt, S.N., Thomson Leighton, F.: A framework for solving VLSI graph layout problems. Journal of Computer and System Sciences 28(2) (1984) 300-343

3. Rosenberg, A.L., Snyder, L.: Bounds on the costs of data encodings. Theory of Computing Systems 12(1) (1978) 9-39

4. Chung, F.R.K.: Labelings of graphs. In Beineke, L.W., Wilson, R.J., eds.: Selected topics in graph theory volume 3. Academic Press (1988) 151-168

5. Hromkovič, J., Müller, V., Sýkora, O., Vrťo, I.: On embedding interconnection networks into rings of processors. Lecture Notes in Computer Science 605 (1992) $51-62$

6. Papadimitriou, C.H.: The NP-completeness of the bandwidth minimization problem. Computing 16(3) (1976) 263-270

7. Harper, L.: Optimal assignment of numbers to vertices. Journal of SIAM 12(1) (1964) 131-135

8. Kuhn, H.W.: The Hungarian method for the assignment problem. Naval Research Logistic Quarterly 2(1) (1955) 83-97 
9. Zhou, S.: Bounding the bandwidths for graphs. Theoretical computer science 249(2) (2002) 357-368

10. de Klerk, E., Eisenberg-Nagy, M., Sotirov, R.: On semidefinite programming bounds for graph bandwidth. Technical report, Centrum Wiskunde \& Informatica, (2011)

11. Yuan, J., Zhou, S.: Optimal labelling of unit interval graphs. Applied Mathematics, A Journal of Chinese Universities 10B(3) (1995) 337-344

12. Lam, P.C.B., Shiu, W.C., Chan, W.H.: Characterization of graphs with equal bandwidth and cyclic bandwidth. Discrete Mathematics 242(3) (2002) 283-289

13. Lam, P.C.B., Shiu, W.C., Chan, W.H.: On bandwidth and cyclic bandwidth of graphs. Ars Combinatoria 47(3) (1997) 147-152

14. Martí, R., Campos, V., Piñana, E.: A branch and bound algorithm for the matrix bandwidth minimization. European Journal of Operational Research 186(2) (2008) 513-528

15. Palubeckis, G., Rubliauskas, D.: A branch-and-bound algorithm for the minimum cut linear arrangement problem. Journal of Combinatorial Optimization (2011) 1-24, DOI: $10.1007 / \mathrm{s} 10878-011-9406-2$ 